\title{
КРИТЕРИИ КЛАССИФИКАЦИИ ТИПОВ КОНТРОЛЬНЫХ ОЦЕНОК ВНУТРЕННЕГО ФИНАНСОВОГО КОНТРОЛЯ
}

\author{
(c) 2021 Петров А. М. \\ доктор экономических наук, профессор Департамента бизнес-аналитики \\ Финансовый университет при Правительстве Российской Федерации, Россия, Москва, \\ E-mail: palmi@inbox.ru
}

Внутренний финансовый контроль - это процесс, осуществляемый менеджерами субъекта хозяйствования с целью получения информации относительно выполнения следующих задач: обеспечения соблюдения законов и нормативных актов; повышения эффективности и рациональности хозяйственной деятельности; формирования достоверной отчетности, а также обеспечения надёжной информацией [3].

Проблемы оценок внутреннего аудита основаны не только на различных концепциях этого термина, существующих на практике, но также являются результатом нерешенных различных критериев классификации для типов контрольных оценок. Оценки, применяемые в контрольной практике, носят различный характер. Это вытекает из специфики управления, которая может проявляться в разных формах и типах, а также превращаться из одной формы в другую. Разнообразие контрольных оценок также связано с тем, что значительная их часть не регламентирована и недостаточно научно-исследована.

Ключевые слова: индикаторы, отрасль, оценка, предприниматель, риски, рынки, ситуация, стратегия, угрозы, управление, экономические субъекты.

В соответствии с формой контроля, оценки представляют собой: оценки предварительного, текущего и последующего контроля. Оценка контроля (включая процесс контроля) посредством проявления трех форм контроля направлена на то, чтобы отразить динамику в развитии финансового контроля, представить изменение поставленных целей и задач, а также требований общества к финансовому контролю. Оценка предварительного контроля представляет обобщенные вопросы в отношении предстоящих целей и задач контроля. На основании этого принимаются решения по устранению выявленных трудностей и проблем. При правильной оценке предварительного контроля его эффективности является наивысшей по сравнению с текущим и последующим контролем, поскольку он дает оценку существующих общих и особых условий, доступных до начала значительных контрольных действий.

Оценка последующего контроля является конкретной с учетом того факта, что объект контроля - это результат уже выполненного действия или процесса. Зачастую определяется путем сравнения результатов предварительного и текущего контроля. Последующий мониторинг представляет собой дальнейшую оценку закон- ности финансовой деятельности контролируемого объекта. На основе оценки вырабатываются рекомендации по улучшению деятельности субъекта хозяйствования.

Текущие оценки зависят от стадии разработки контролируемого объекта, а также от обобщенной информации, доступной контроллеру. В отличие от промежуточных и итоговых контрольных оценок, которые определяются точным формальным требованием (внутренним приказом, указом, постановлением, стандартом, законом и т.д.). Текущие контрольные оценки должны разрабатываться в ходе текущего процесса контроля (Taylor and Glezen, 1991) [9]. Мы считаем, что точное определение промежуточных и итоговых контрольных оценок напрямую связано с проводимыми оценками. Сопоставимость данных, показателей и факторов не может быть достигнута, если факты, процессы и события не будут обобщены и оценены заранее.

В зависимости от характера поставленных задач контрольные оценки отличаются из-за существенных различий в контрольных задачах. Задачи ВФК направлены на контролируемый объект, а именно: на охрану имущества субъекта хозяйствования; оптимизацию доходов и минимизацию расходов; достижение эффективно- 
сти производственных мощностей и т.д. Задачи управления соответствуют комплексному, постоянному и оперативному характеру внутреннего финансового контроля.

Следующий критерий, по которому могут быть классифицированы контрольные оценки, связан с принадлежностью оценщика. Оценщики могут быть как внешними по отношению к субъекту хозяйствования, так и внутренними. Таким образом, и контрольные оценки могут быть внутренними и внешними для субъекта хозяйствования. Вопрос о методологии оценки внешнего и внутреннего контроля все еще остается проблематичным.

Традиционно аудиторы применяют качественные методы, такие как вопросники, списки, диаграммы, интервью, а также дополнительные методы и ролевые игры. Выбор каждого метода зависит от заранее поставленных целей и задач контроля. Применение качественных методов требует хорошей профессиональной подготовки аудиторов. Качественные методы являются предпочтительными в практике контроля изза их универсальности и широко применяемых инструментов, и, с другой стороны, они могут использоваться для выполнения множества задач, поскольку затраты и время на реализацию их незначительны.

Оценки контроля качества основаны на наблюдениях, а количественные оценки - на количественных обследованиях наблюдаемого объекта. Количественные исследования проводятся с использованием различных научных методов, которые включают: сбор эмпирических данных; моделирование и анализ данных; осуществление экспериментального контроля; порождая разные теории и гипотезы.

В соответствии с предметом оценки контрольные оценки нацелены на все возможные объекты субъекта хозяйствования, которые входят в сферу контроля, такие как: измерение эффективного использования и ввода запасов; оценка квалификации персонала; измерение состояния и изменений в активах субъекта хозяйствования; оценка состояния системы внутреннего контроля и состояния системы финансового управления и контроля в государственном секторе; оценка ИТ-ресурсов субъекта хозяйствования; оценка общей структуры субъекта хозяйствования; оценка имеющихся ресурсов для реализации конкретного инвестиционного проекта и т.д. После того, как на контролируе- мом объекте были проведены конкретные процедуры контроля, контролеры (т.е. инспекторы, внутренние или внешние аудиторы) должны предоставить экспертное заключение, заканчивающееся оценкой состояния контролируемого объекта, с рекомендациями и возможностями для улучшения этого состояния. В случае отклонения от контрольных норм, соответствующая система действий также должна быть предложена руководителям субъекта хозяйствования.

Внутренний аудит, как часть контроля субъекта хозяйствования, также подлежит оценке и определению уровня качества, которого внутренние аудиторы хотели бы достичь и / или достигли в своей работе. Практика контроля позволяет при генерации окончательной оценки состояния контроля в субъекте хозяйствования также представлять оценку состояния внутреннего аудита для тех субъектов, которые имеют утвержденный отдел внутреннего аудита. В связи с этим мы предполагаем, что критерии, по которым могут быть классифицированы оценки внутреннего аудита, основаны на четырех основных характеристиках.

Во-первых, в соответствии с местом, где были сделаны оценки, и в соответствии с принадлежностью оценщиков к самому субъекту хозяйствования, оценки являются внутренними и внешними. Внутренние оценки включают в себя постоянные проверки работы отдела внутреннего аудита и периодические проверки, проводимые путем самооценки или оценки другими лицами, знакомыми с практикой внутреннего аудита и внутреннего контроля. Стандарты соответствуют требованиям ст. 19 Закона о внутреннем аудите государственного сектора. Внутренние оценки могут проводиться руководящими органами субъекта хозяйствования, руководителями подразделений внутреннего аудита, а также самими внутренними аудиторами. Внешние оценки осуществляются не реже одного раза в 5 лет людьми, находящимися за пределами субъекта хозяйствования и имеющими сертификат «внутреннего аудитора в государственном секторе» или действующий международный сертификат внутреннего аудитора.

Второй критерий для классификации оценок - согласно проверке, выполненной аудиторами. Обусловленные этой особенностью, оценки могут быть текущими и периодическими. Текущие оценки основаны на постоянном рассмотрении всего спектра аудиторской деятель- 
ности. В свою очередь, текущий обзор включает в себя: мониторинг аудиторских обязательств; обратная связь между руководителем службы внутреннего аудита и аудиторского процесса и анализом показателей эффективности аудита. Текущие оценки формируются на основе текущего состояния деятельности внутреннего аудита субъекта хозяйствования. Периодические оценки основаны на периодическом обзоре аудиторской деятельности. Периодические проверки охватывают деятельность внутреннего аудита как минимум в течение одного календарного года, в отличие от текущих проверок, где можно выполнить оценку состояния внутреннего аудита текущего дня, недели или месяца. Периодические обзоры включают в себя: самооценку; внутренние проверки аудиторской деятельности, которые находятся в ведении внутренних аудиторов, и собеседования для расследования проверенных структурных подразделений субъекта хозяйствования.

Третий критерий определяется в зависимости от объекта оценки. По сути, аудит соответствия - это «проверка систем финансового управления и контроля, включая внутренние аудиторские и управленческие решения, касающиеся субъекта хозяйствования, планирования, управления, отчетности и контроля бюджетных и других государственных ресурсов, а также деятельности проверяемого субъекта с целью соблюдения требований». Согласно объекту, оценки могут проводиться при измерении качества процедур внутреннего аудита. В свою очередь, контроль качества процедур внутреннего аудита - это набор хорошо зарекомендовавших себя мер и процедур, выполняемых внутри самих процессов. Целью этого последующего контроля является обеспечение качества внутреннего финансового контроля и подтверждение эффективности и результативности рассчитанных результатов контролера.

Вопрос контроля качества всегда был фундаментальным и проблематичным для государственного сектора. В современных экономических условиях задача внутреннего финансового контроля обуславливается не только тем, чтобы повысить ценность субъекта хозяйствования, но и оправдать ожидания заинтересованных сторон. Это также вызывает необходимость определения того, насколько качественно выполняются процессы внутреннего финансового контроля и соответствуют ожиданиям ряда заинтересованных сторон (государственных департаментов, учреждений, субъектов хозяйствования и т.д.). В этом отношении согласно международным стандартам профессиональной деятельности «внутренний аудит» основывается на принципах, применяемых в деятельности аудируемых субъектов хозяйствования в соответствии с рядом правовых и нормативных требований. Они представляют концептуальную основу, в которой выполняется процесс внутреннего аудита, чтобы он соответствовал критериям качества.

\section{Библиографический список}

1. Федеральный закон от 26.07.2019 № 199-Ф3 «О внесении изменений в Бюджетный кодекс Российской Федерации в части совершенствования государственного (муниципального) финансового контроля, внутреннего финансового контроля и внутреннего финансового аудита» // СПС КонсультантПлюс.

2. Батуева, А.Б. Система внутреннего контроля / А. Б. Батуева // Молодой ученый.-2018. - № 16 (202).- С. $148-150$.

3. Булыга, Р.П.Классификация и стандартизация финансового контроля и аудита в российской федерации // Учет. Анализ. Аудит. - 2017.- № 5.- С. 10-17.

4. Завьялова, Л.В., Шилехин, К.Е. Внутренний финансовый контроль в условиях нового законодательства // Вестник Омского университета. Серия «Экономика».-2013.- № 4.- С. 88-99.

5. Кучеров, А.В. Внутренний аудит в России / А.В.Кучеров, Е.В.Леушина.- Текст: непосредственный, электронный // Молодой ученый. - 2013. - № 6 (53). - C. 359-362. - URL: https://moluch.ru/archive/53/7029/.

6. Петров А. М., Бурцева К. Ю. К вопросу организации внутреннего финансового контроля в рамках расходования средств на федеральные проекты // Экономические науки. - 2020. - № 188. - С. 113-119.

7. Петров, А. М. Оперативный и превентивный инструментарий реализации внутреннего финансового контроля в рамках расходования средств на федеральные проекты // Экономические науки. - 2020.- № 185.С. 169-178.

8. Саввина, О.В. О современной модели финансового надзора // Финансы и кредит. - 2015.- № 34 (658).- С. 47-57.

9. Donald H. Taylor, G. William Glezen / Auditing: Integrated Concepts and Procedures. - Wiley, 1991. 
10. Nedyalkova P. Quality of Internal Auditing in the Public Sector Perspectives from the Bulgarian and International Context Univ. of Econ. - Varna, 2019, pp. 266.

11. Chernysheva N.A., Perskaya V.V., Petrov A. M., Bakulina A. A. GREEN ENERGY FOR BELT AND ROAD INITIATIVE: ECONOMIC ASPECTS TODAY AND IN THE FUTURE / International Journal of Energy Economics and Policy. 2019. T. 9. № 5. C. 178-185.

12. Kevorkova Z.A., Petrov A. M., Savina N. V. TOWARDS LIABILITIES OF CORPORATE SYSTEMS / International Journal of Civil Engineering and Technology. 2019. T. 10. № 2. C. 1582-1593.

13. Petrov A.M., NikiforovaE.V., Kiseleva N.P., GrishkinaS.N., Lihtarova O. V. CREATION OF THE REPORTING ON SUSTAINABLE DEVELOPMENT OF COMPANIES BASED ON SOCIOECONOMIC MEASUREMENT STATISTICS / International Journal of Recent Technology and Engineering. 2019. T. 8. № 2. C. 4005-4012.

14. SotnikovaL.V., PolenovaS.N., MislavskayaN.A., PetrovA.M., BasovaM.M. SUSTAINABLE DEVELOPMENT, MACRO AND MICRO LEVEL: RUSSIAN AND FOREIGN MODEL / International Journal of Recent Technology and Engineering. 2019. T. 8. № 2. C. 4524-4532.

15. Kosolapova M.V., Muravitskaya N.K., Tolmachev M.N., MelnikovaL.A., PetrovA.M. TECHNOLOGY FOR SOLVING THE PROBLEMS RELATED TO THE IMPLEMENTATION OF THE CONCEPT OF PRESERVING CAPITAL IN ACCOUNTING AND STATISTICS / International Journal of Recent Technology and Engineering. 2019. T. 8. № 3. C. 789-792.

16. Kosolapova M.V., PetrovA.M., YshanovI.G., Muravitskaya N.K., NurmuhamedovaH.S. THE ECONOMIC SIGNIFICANCE OF STATISTICAL RESEARCH ACTIVITIES OF REPRESENTATIVE OFFICES OF COMPANIES ABROAD / International Journal of Innovative Technology and Exploring Engineering. 2019. T. 8. № 10. C. 27132722.

17. Petrov A.M., Yurasova I. O., Putihin Y. E., Poluleh M. V., Erohina V.N. ACCOUNTANT MODELING TECHNOLOGY AND STATISTICS IN THE CONTEXT OF THE NEW EDUCATIONAL CONCEPT / International Journal of Innovative Technology and Exploring Engineering. 2019. T. 8. № 12. C. 3214-3217.

18. Petrov A.M., Kiseleva N.P., KevorkovaZ.A., Melnikova L.A., YshanovI.G. PRESENT DEVELOPMENT PRACTICES FOR TAX, FINANCIAL AND STATISTICAL REPORTING IN THE RUSSIAN FEDERATION / International Journal of Innovative Technology and Exploring Engineering. 2019. T. 8. № 12. C. 3538-3542.

19. Karpova T.P., Petrov A.M., Antonova O. V. DIRECTIONS OF ACCOUNTING DEVELOPMENT IN THE CONDITIONS OF DIGITALIZATION / Journal of Advanced Research in Dynamical and Control Systems. 2018. T. 10. № 7 Special Issue. C. 117-125.

20. Lymar M.P., Kevorkova Z. A., Petrov A. M.THE CONVERGENCE OF NATIONAL AND INTERNATIONAL ACCOUNTING STANDARDS: CHINESE EXPERIENCE / International Journal of Civil Engineering and Technology. 2018. T. 9. № 13. C. $82-94$. 